Poul Erik Jфrgensen*

\title{
Gaining Compliance in Pressure Politics: An Inter- disciplinary Study of Requestive Message Produc- tion in the British Political Consultancy Industry
}

\section{Introduction}

The gaining of compliance is unquestionably among the most important acts of human communication insofar as its performance may determine the future of an interpersonal relationship. Just consider the police officer attempting to persuade demonstrators to disband peacefully, the doctor trying to dissuade a patient from smoking, or the married couple having a heated argument about the distribution of household chores. Not surprisingly, the significance of persuasion to most interpersonal exchanges, private or professional, has caused scientists in various research fields to investigate the nature of requestive behaviour at all levels from macro to micro.

In some communication research, the focus is moved from the verbal encounters of naturally occurring situations to the carefully planned production of persuasive messages characteristic of, for example, written business communication. The correct and effective delivery of requests is a recurring and perhaps even the most central activity of professionals producing discourse intended for consumption in a business environment. When business partners exchange written messages, their main objective is usually to induce a change in either the behaviour, the attitudes, or the opinions of one another. In other words, a business operator's principal concern is nearly always to gain the compliance of the recipient of the message in furtherance of a particular objective.

* Poul Erik Jørgensen

Engelsk Institut

Handelshøjskolen i Århus

Fuglesangs Allé 4

DK-8210 Arhus V 
Increasingly, business organisations are also recognising a need to influence political decision-making. This happens at all levels of government in response to the now pivotal role played by the executive branch of modern welfare states as regulator and single-largest customer of private business and industry. Some private concerns employ in-house staff to manage their growing contact with political decision-makers while others prefer to purchase the services of firms of political consultants whose main assets are their first-hand experience of the political process and their ability to professionally handle this specialised form of communication.

\section{Background}

The thesis is concerned with how business organisations go about producing requestive messages specifically targeting government ministers and high-ranking civil servants through a multi-phased examination of the requestive discourse practices of the political consultants who provide business clients with expert advice. The British political consultancy industry lends itself ideally to this purpose insofar as it is a young industry whose inception can be traced with fairly great precision. By some analyses, former prime minister Margaret Thatcher happened to unwittingly prepare the ground for a highly professionalised consultancy industry from the moment she was installed in political office in 1979 by declaring that she would not accept obstruction from constitutional outsiders. Thatcher's adamant opposition to interference from outside players was supposedly caused by the bitter experiences of the Conservative and Labour governments of the 1970s (see e.g. Baggott 1995, Peele 1995). To this, Bill Jones and Dennis Kavanagh (1994:216) comment that "her style ... was to squeeze pressure groups towards the periphery of decisionmaking, to be less interested in listening and more concerned to act in accordance with what has already been decided as necessary". This apparently abrupt dismissal of several of the most powerful pressure groups from direct negotiations with the government of the day appears to have induced the entire field of pressure groups to explore alternative avenues to political influence with greater vigour. The advent of Margaret Thatcher thus marks a disruption in the relationship with political decision-makers for large parts of British trade and industry which then, by necessity, came to rely on political consultancy as a new instrument 
for seeking influence. With Tony Blair's New Labour, this relatively young industry appears to have retained a strong foothold in the market for communication services, and following the 1997 general election victory a substantial number of young Labourites, who had been staffing the Blair campaign office, obtained employment with several of the leading UK firms of political consultants.

For a given influence attempt, the consultancy will identify all the relevant key people in the Government, in the higher civil service, on committees and quangoes, in the political parties, and in Parliament. The consultancy will then analyse the positions taken by these core audiences, will look for cleavages and alliances between them, will establish one or more achievable objectives, and will subsequently define a strategy that is likely to produce a positive response to the requests made. On the basis of such data, the influence campaign is likely to be designed to target several of the key players to obtain the best possible impact, and the first contact is usually by means of a written inquiry. As pointed out by Yli-Jokipii (1994:48), Britain essentially remains a "writing society", and all first contacts in weighty matters may be expected to be by way of a letter, even if the interactants already have an established relationship. So, the letter remains the standard opening move in the majority of influence attempts in which a business venture contacts either an MP or a top-level decision-maker within the executive branch. The letter will always be signed by the business client and it will not be immediately apparent that it was produced through the agency of a political consultant. One of the consultancy directors interviewed during the course of the study summed the consultancy's function up in this way: "All that we're doing with our clients is helping them to put their case, and it is a case of knowing who they've got to talk to, when they've got to go and talk to them, and what they've got to say. And all you do is you put the case at the best time, in the best way, and keep your fingers crossed that it is listened to".

\section{Model}

The central aim of the project was (a) to determine if professional communicators of the highest order are methodically stringing together the options available to them across the various phases of the message production process, and (b) if their choices link together in a patterned way. 
A third objective for the study was to examine how the actual production of persuasive messages might be affected by the message producer's perception of the situational and relational circumstances most salient to the case at hand. For this purpose, the following overarching model combining a collection of macro resources from both communication and linguistic theory was suggested in order to arrive at a comprehensive description of what shapes the final product of a requestive event.

The model is based on the Goals - Planning - Action sequence (GPA sequence) which has inspired researchers in various disciplines concerned with human behaviour and tactical communication (see e.g. Dillard 1990a). The model presupposes a "producer perspective" and contains three distinctly different phases which together constitute the message production process. The phases form a sequential process moving from left to right with the choices made in the two first phases being extratextual and thus preparing the ground for the textual choices to be made in the third and final phase. The model assumes that the message producer first decides what to achieve with the message (instrumental goals), then chooses the rhetorical tactics expected to be most effective in delivering the desired outcome (planning), before finally converting these intentions and tactical choices into a discursive format (action). Importantly, the choices made in the Goals phase are taken to be influenced by a variety of situational and relational assessments which are representative of the whole arsenal of contextual information that the message producer can access and bring to bear upon the message production process. 
Motive, political context, transactional stage, role relationship

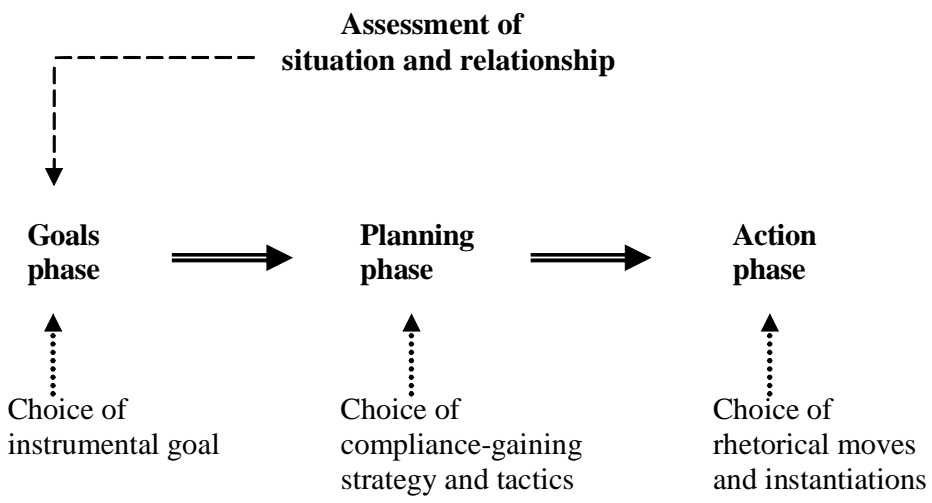

Model: GPA message production sequence (producer perspective).

Each of the phases of the GPA sequence has been thoroughly accounted for independently of each other. But there appears to be no fully fledged theory which speculates that the three phases can be defined at the same level of abstraction, that they might link together, and that they might link together in a patterned way. Thus, for example, Tracy and Coupland (1990:8) write that "it is far from a simple matter how a speaker gets from the general strategy level - the level at which people typically report (e.g. I'd be friendly and then ask for the favour) to the specifics of talk". Likewise, it remains unclear if and how the message producer's perception of situational and relational circumstances affects the three not only sequentially but also dynamically related phases. To account for the contextual impact together with the two extra-textual phases, the study draws on so-called compliance-gaining theory which has developed into a dominant tradition in interpersonal communication research over the past twenty-five years. For the treatment of the textual phase, functional elements of linguistic genre theory, as powerfully formulated by Swales (1990) and Bhatia (1993), have been incorporated and expanded upon. In other words, a primary challenge for the study was one of hooking together compatible macro-level components in an interdisciplinary fash- 


\section{8}

ion to test the validity of the GPA formula when applied to the written medium in a professional setting.

\section{Method}

Various attempts have been made by different researchers over the years to identify and classify a small number of instrumental goals with the common feature of being specifically relevant to persuasive communication (see e.g. Schank and Abelson 1977). For the current study, nine mutually exclusive instrumental goals (elicit support, enforce obligation, share activities, gain assistance, obtain permission, change opinion, change relationship, give advice, explain breach) were derived on the basis of the synthesised typological framework offered by Cody et al. (1994) and were subsequently classified into four overriding goal type categories.

To operationalise the Planning phase, the tactics typology scheme advanced by Wiseman and Schenck-Hamlin (1981) and later elaborated on by Tracy et al. (1984) was preferred to a range of similar schemes on the grounds that it is inductively derived and is expressed in a nonrestrictive format that allows it to be immediately applied to discourse. The scheme was however slightly modified to obtain a framework tailored precisely to the real-life professional discourse collected for analysis. The typology consists of eleven basic tactics (ingratiation, promise, debt, esteem, allurement, threat, guilt, warning, altruism, simple request and explanation) which were ordered into three different main categories and four subcategories to facilitate analysis on the basis of tactics type. The message producer may use any number of these tactics to communicate the instrumental goals chosen for the message, and each of these primary or core tactics may be supported by any combination of tactics that work towards the same end (tactics expansions).

The string of tactics chosen for any one message thus constitutes an extra-textual message strategy which the message producer can utilise for the encoding process that subsequently occurs at the textual macrolevel. At this level, persuasive messages can be arranged into two primary move types (legitimising and requestive moves) and two secondary move types (facilitative and concluding moves). The compliance-gaining tactics always come within one of the primary move types, each of which is represented by three different moves (legitimising moves: (a) explaining 
position, (b) defending position, (c) explaining situation; requestive moves: (a) suggesting activity, (b) claiming support, (c) soliciting response). These moves, which were derived empirically, can be enacted in a variety of ways. Within the present framework it is suggested that, together, the moves cover twenty-three different instantiations. For example, the move "explaining position" can be instantiated by (a) offering an opinion, (b) presenting an ambition, (c) advocating a solution, (d) expressing support, or (e) expressing concern. The intention was to suggest a small and consequently manageable set of mutually exclusive moves that can impart compositional value to the chosen instrumental goals and compliance-gaining tactics.

To decide why one instrumental goal was chosen over another, it is necessary to consider a range of situational and relational dimensions external to the GPA sequence. By the mid-1970s, scholars of communication thus began investigations to capture a global set of contextual perception factors to represent the cognitive load generated in any requestive situation in addition to that which could be attributed to the psychological make-up of the individual message producer. In spite of these considerable efforts, Miller et al. (1994:190) point out that too little is still known about the diversity of contextual dimensions, about how these dimensions influence each other, and about how they influence message production choices. Taking inspiration from Cody and McLaughlin (1980), Cody et al. (1994) and Tracy et al. (1984), an attempt was made in this study to determine the impact of the situational context through an assessment of the transactional stage, the local context and the global context. As part of this attempt, the relational context was also evaluated by assessing a set of six different factors which capture different aspects of the role relationship (intimacy, dominance, resistance, personal benefits, relational consequences, rights).

\section{Procedure}

Three leading UK political consultancies agreed to participate in a series of interviews and to supply the necessary letters for the research corpus. First, a senior director from each of the consultancies was invited to describe the consultancy's corporate culture, how the consultancy is organised, how it operates, and the services offered to business clients. 
In the second round of interviews, eight different political consultants were asked a set of questions for each of the phases of the GPA sequence and for the impact of contextual perceptions on the Goals phase. Each consultant was then asked to submit for analysis a number of standardlength requestive letters produced on behalf of a business client and addressed to either a private member of Parliament, a senior government minister, or a senior official in the higher civil service. The letters were subsequently assessed for situational and relational impact before they were analysed for choice of instrumental goals, compliance-gaining tactics and strategies, moves and their instantiations. Finally, a third round of interviews was conducted to determine the extent to which the eight consultants did agree with or would challenge the findings of the corpus analysis.

The purpose of the second and third rounds of interviews was to create a background against which to compare the results of the message analysis in order to avoid committing the post hoc fallacy of concluding "that it is raining simply because people are carrying umbrellas", as warned against by Dillard (1990b:70-71), and in order to meet the requirements to informant verification outlined by Bhatia (1993:34).

\section{Results and discussion}

The analysis of the letter corpus furnishes a bottom-up account allowing contemplation of the two main research questions: (1) are the three phases of the GPA sequence in fact compatible at a macro-level of abstraction and, if so, (2) do the phases actually interface in a patterned way. In addition, the analysis provides data to allow a tentative consideration of the third research question of how the message production sequence is influenced by situational and relational perceptions.

The empirical analysis showed that once the motive or requestive force of the influence attempt is established, it is relatively simple to identify the instrumental goals of a letter. Once the textual passages containing the goals were identified, it was also a fairly straightforward job to spot the compliance-gaining tactics and the corresponding moves and instantiations within the relevant passages. The requestive utterances of the corpus letters were thus examined in three individual stages for these features, and the results were juxtaposed to create a full picture of each individual utterance. To exemplify this, the following utterance has a 
declarative form but may still be picked up by the message receiver as a request because of the content words "helpful" and "feel able to". The instrumental request goal is to "elicit the message receiver's support" by "asking him/her to assist in persuading a third party". The message producer pursues this goal by making an "altruistic" appeal for help (tactic). This, in turn, is done by "suggesting an activity" (move) consisting in a "proposal for an action" (instantiation) to be taken by the message receiver. These details, it is suggested, can all be derived from the same utterance.

(1) It would be very helpful if you felt able to ask the Minister to consider this matter.

The GPA relationships thus elicited from this utterance can be depicted in the following way:

\begin{tabular}{|ll|l|ll|}
\hline Instrumental goal & Goal task & Tactic & Move & Instantiation \\
$\begin{array}{l}\text { Elicit } \\
\text { support }\end{array}$ & $\begin{array}{l}\text { Ask receiver to assist in } \\
\text { persuading third party }\end{array}$ & Altruism & Suggesting & Proposing action \\
& & & activity & \\
\hline
\end{tabular}

This requestive core will invariably be preceded or followed by a number of supporting passages in which the message producer uses an appropriate number of tactics expansions which are, in turn, rhetorically enacted via a selection of moves and their instantiations. This might, for example, result in the following expanded message production profile:

\begin{tabular}{|c|c|c|c|c|}
\hline \multirow[t]{4}{*}{ Instrumental goal } & Goal task & Tactics/expansions & Moves & Instantiations \\
\hline & & Ingration & $\begin{array}{l}\text { Explaining } \\
\text { position }\end{array}$ & $\begin{array}{l}\text { Expressing } \\
\text { support }\end{array}$ \\
\hline & & Guilt & $\begin{array}{l}\text { Explaining } \\
\text { position }\end{array}$ & $\begin{array}{l}\text { Expressing } \\
\text { concern }\end{array}$ \\
\hline & & Explanation & $\begin{array}{l}\text { Defending } \\
\text { position }\end{array}$ & $\begin{array}{l}\text { Listing } \\
\text { support(ers) }\end{array}$ \\
\hline $\begin{array}{l}\text { Elicit } \\
\text { support }\end{array}$ & $\begin{array}{l}\text { Ask receiver to } \\
\text { assist in persuading } \\
\text { third party }\end{array}$ & Altruism & $\begin{array}{l}\text { Suggesting } \\
\text { activity }\end{array}$ & Proposing action \\
\hline
\end{tabular}


Crucially, no gaps or inconsistencies appeared between the applied typologies during the course of the analysis, which meant that the interfacing process exemplified above could be replicated across the corpus in support of the relative logic that the sequential GPA relationships can be determined when the phases are filled with compatible entities at the same level of abstraction.

However, the real-life data did not confirm the assumption that a particular goal choice would either prescribe a particular use of tactics or guide message producers towards two or more suitable options. Rather, the data show that, with unfaltering care, the political consultants in this study choose the tactic "simple request" for core tactic irrespective of their choice of instrumental goals. The exact same observation was made with respect to their choice of the tactics expansions incorporated in the messages to pave the way for the core requestive passages. Since there was an overwhelming preference for the tactics expansions "ingratiation" and "explanation", the expectation that message producers will opt for forceful or complex strings of tactics and tactics expansions in combination with particular goals was thus not borne out by the data. So, it appears that the tactics used for requestive messages combine into rhetorical strategies that are unaffected by goal choice and must therefore be decided by the message producers on the basis of contextual deliberations.

Likewise, there was no evidence of a significant one-to-one relationship between instrumental goals and requestive moves and their instantiations in the Action phase. So, for example, it is not as if the operational goal "gain assistance" automatically calls for the requestive move "suggesting activity" and one of its instantiations, say, "proposing action". While goal choice is not prescriptive in this way, it nevertheless appears to guide the choice of requestive moves and their instantiations insofar as only a restricted number of instantiations seem suited for each individual goal. With the message producers' clear preference for the requestive move "suggesting activity", irrespective of their choice of goal, there is strong indication that goals alone do not determine which of the requestive move options, or, in turn, which of several possible instantiations of a move, is the best suited in the given circumstances.

Since the tactic "simple request" is used with such great frequency in the corpus, no clear patterns were found to emerge from the relationship between tactics in the requestive passages and the matching instantiations 
of requestive moves either. However, the expected strong correlations between the tactics expansions used in the supportive passages ("ingratiation" and "explanation") and their matching legitimising moves ("expressing support", "offering opinion/analysis", and "outlining background") were obtained. In the same way as goal choice was found to at least guide the choice of instantiations of the requestive moves, tactics expansions thus appear to also guide the choice of instantiations of the legitimising moves. Therefore, the data do not convincingly show tactics expansions in the supportive passages to carry clear and final instructions on which is the most suitable move or instantiation for representing them. As with goals and tactics, then, the final choice between two or more instantiations suited for the same tactics expansion is again, by default, taken to be conditioned by relational and situational factors.

The interviews conducted with the participating political consultants prior and subsequent to the corpus analysis provided a solid platform for assessing the results derived in a bottom-up fashion. Interestingly, and in contrast to results obtained from their letters, the consultants indicated that they believe themselves to be using both the goal and the tactics repertoires to their full potential, even though they later testify to be veering on the side of caution when addressing political decision-makers. The interviews also provided the necessary understanding of how the consultants perceive the situations and relations creating the contextual background of each message in the corpus. While this information was valuable in creating complete profiles for each letter, it did not provide evidence for the entire corpus to suggest that a particular combination of contextual factors either prescribes a particular goal choice or guides message producers towards a limited number of goal options. In other words, the initial assumption that, in the specialised context of professional discourse production, individual situational and relational assessments can be summed up to give an accumulated result determining goal choice could not be substantiated. This finding is in harmony with the results of previous research (see Cody et al. 1986) demonstrating that while there may be patterns in the relationship between individual contextual factors and tactics choice, such patterns are eroded when clusters of contextual factors are applied. 
This leads to the overall conclusion that, in the production of written messages, instrumental goal choice, planning and rhetorical action form a sequential relationship in which the three phases can be defined and linked at the same level of abstraction. However, the sequence is only partially dynamic in the sense that goal choice seems to guide message producers in their choice of moves and instantiations, but not in their choice of compliance-gaining tactics or tactics strategies. This was however shown to be the case in studies by Cody et al. (1994:50-55) and other communication theorists, at least with regard to the issuing of requests in the spoken medium. Also, the current research effort only gave evidence of a guiding effect of tactics expansions on the choice of moves and instantiations. The dynamic relationship between goals, core tactics and rhetorical action could not be established because of the message producers" propensity to use the tactic "simple request". In other words, only weak confirmation was obtained of various researchers' claim that "influence goal type affects planning and message output", and that "failure to attend to variations at the beginning of the GPA sequence (i.e. to different goal types) is bound to obfuscate potentially important variations in later phases of the sequence (i.e. planning and action)" (Dillard 1990a:46).

Since the contextual influence is exterior to the GPA sequence, only a tentative attempt was made to show how the situational and relational context impacts on message producers' choice of instrumental goals. For that purpose, a provisional framework of situational and relational perception factors was incorporated into the analysis of the real-life messages constituting the authentic twenty letter corpus. However, the interpersonal power quality derived for the individual letters from a balanced assessment of the set of relational perception factors proved to be a poor indicator of instrumental goal choice. Likewise, the three situational factors of motive, transactional stage, and local and global context afforded no reliable evidence as to why message producers arrive at particular goal choices.

\section{References}

Baggott, Rob (1995). Pressure Groups Today. Manchester University Press, Manchester. Bhatia, Vijay K. (1993). Analysing Genre: Language Use in Professional Settings. Longman, London. 
Cody, Michael J. \& McLaughlin, Margaret L. (1980). Perceptions of Compliance-Gaining Situations: A Dimensional Analysis. Communication Monographs 47. 132-148.

Cody, MichaelJ., Greene, John O., Maston, Peter J., O’Hair, H. Dan, Baaske, Kevin T. \& Schneider, Michael J. (1986). Situation Perception and Message Strategy Selection. In Margaret L. McLaughlin (ed.), Communication Yearbook 9. 390-420. Sage, Beverly Hills, CA.

Cody, Michael J., Canary, Daniel J. \& Smith, Sandi W. (1994). Compliance-Gaining Goals: An Inductive Analysis of Actor's Goal Types, Strategies and Successes. In John A. Daly \& John M. Wiemann (eds.), Strategic Interpersonal Communication. 33-90. Lawrence Erlbaum, Hillsdale, NJ.

Dillard, James P. (1990a). A Goal-Driven Model of Interpersonal Influence. In James P. Dillard (ed.), Seeking Compliance: The Production of Interpersonal Influence Messages. 41-56. Gorsuch Scarisbrick, Scottsdale, AZ.

Dillard, James P. (1990b). The Nature and Substance of Goals in Tactical Communication. In Michael J. Cody \& Margaret L. McLaughlin (eds.), The Psychology of Tactical Communication. 70-90. Multilingual Matters, Clevedon.

Jones, Bill \& Kavanagh, Dennis (1994). British Politics Today. Manchester University Press, Manchester.

Miller, Lynn Carol, Cody, Michael J. \& McLaughlin, Margaret L (1994). In Mark L. Knapp \& Gerald R. Miller (eds.), Handbook of Interpersonal Communication. 162198. Sage, London.

Peele, Gillian (1995). Governing the UK. Blackwell Publishers, Oxford.

Schank, Roger C. \& Abelson, Robert P. (1977). Scripts, Plans, Goals and Understanding: An Inquiry into Human Knowledge Structures. Lawrence Erlbaum, Hillsdale, NJ.

Swales, John M. (1990). Genre Analysis: English in Academic and Research Settings. Cambridge University Press, Cambridge.

Tracy, Karen, Craig, Robert T., Smith, Martin \& Spisak, Frances (1984). The Discourse of Requests: Assessment of a Compliance-Gaining Approach. In Human Communication Research 10. 513-538.

Tracy, Karen \& Coupland, Nikolas (1990). Multiple Goals in Discourse: An Overview of Issues. In Karen Tracy \& Nikolas Coupland Nikolas (eds.), Multiple Goals in Discourse 1-13. Multilingual Matters, Clevedon

Wiseman, Richard L. \& Schenck-Hamlin, William (1981). “A Multidimensional Scaling Validation of an Inductively-Derived Set of Compliance-Gaining Strategies". Communication Monograps 48. 251-270.

Yli-Jokipii, Hilkka (1994). Requests in Professional Discourse: A Cross-Cultural Study of British, American and Finnish Business Writing. Suomalainen Tiedeakatemia, Helsinki 
